\title{
APLICACIÓN CONTABLE DEL VALOR PRESENTE EN EL CONTEXTO VENEZOLANO
}

\author{
HEIBERG ANDRÉS CASTELLANOS SÁNCHEZ** \\ UNIVERSIDAD DE LOS ANDES, MÉRIDA - VENEZUELA
}

Recibido/ Received/ Recebido: 04/11/2013 - Aceptado/ Accepted/ Aprovado: 06/05/2014

\begin{abstract}
Resumen
La NIIF 13 (2012) "medición del valor razonable" está orientada a unificar, en un único estándar, toda la normativa existente acerca de las mediciones realizadas a valor razonable. En este contexto normativo, el valor presente, criterio valorativo perteneciente a las variables del nivel 3 de la jerarquía, se ha erigido como la técnica financiera de mayor aceptación tanto en el ámbito de la práctica profesional como en el de los organismos emisores. Este documento se centra en dilucidar los criterios utilizados por los contadores públicos en la aplicación de la técnica de valor presente en el contexto venezolano. El estudio se enmarca dentro de un enfoque cualitativo, circunscrito a la modalidad documental, apoyado en la opinión de expertos. Se concluye que la herramienta financiera más utilizada es la técnica del valor presente esperado en su modalidad de método 2 , para cuya construcción se emplea el "modelo de valuación de activos de capital" a fin de obtener la tasa de descuento.
\end{abstract}

Palabras clave: Valor presente, valor razonable, NIIF 13, flujos de efectivo futuros, tasa de descuento.

\section{ACCOUNTING IMPLEMENTATION OF THE PRESENT VALUE IN VENEZUELAN CONTEXT*}

\begin{abstract}
IFRS 13 (2012) "Fair value measurement" is aimed to unify, in a single standard, all existing rules about fair value measurements. In this regulatory context, the present value, evaluative criteria of Level 3 hierarchy varables, has emerged as the most widely accepted financial technique both in professional field practice and issuing authority. This paper focuses on elucidating the criteria used by public accountants of present value technique implementation in Venezuelan context. The study is part of a qualitative approach, based on secondary information supported by expert's opinion. It is concluded that the most common financial tool used, is Method-2 expected present value technique, for its construction the "capital asset pricing model" is used to get the discount rate.
\end{abstract}

Keywords: Present Value, Reasonable Value, IFRS 13, Future Cash Flows, Discount Rate.

Este artículo de investigación científica corresponde a la evaluación final del Seminario Doctoral "Investigación Libre I" asignatura adscrita al Doctorado en Ciencias Contables de la Universidad de los Andes, Mérida - Venezuela.

* Profesor de Planta del Departamento de Contabilidad y Finanzas de la Universidad de los Andes (ULA), Mérida. Jefe de la Cátedra de Contabilidad Superior de la ULA. Integrante del Grupo de Investigación en Finanzas, Auditoría, Contabilidad y Epistemología (GIFACE) del Postgrado en Ciencias Contables de la ULA. Participante de la Sexta Cohorte del Doctorado en Ciencias Contables de la ULA. Magíster en Ciencias Contables, Licenciado en Contaduría Pública, Licenciado en Administración egresado de la ULA. Correo electrónico: heiberg@ula.ve 


\title{
APLICAÇÃO CONTÁBIL DO VALOR PRESENTE NO CONTEXTO VENEZUELANO
}

\begin{abstract}
Resumo
A NIIF 13 (2012), "medição do valor razoável", está orientada a unificar, num único padrão, todo o regulamento existente a respeito das medições realizadas a valor razoável. Neste contexto normativo, o valor presente, critério valorativo pertencente às variáveis de nível 3 da hierarquia, erigiu-se como a técnica financeira de maior aceitação tanto no âmbito da prática profissional como no dos organismos emissores. Este documento se centra em elucidar os critérios utilizados pelos contadores públicos na aplicação da técnica de valor presente no contexto venezuelano. $\mathrm{O}$ estudo se emoldura dentro de um enfoque qualitativo, circunscrito à modalidade documental, apoiado na opinião de peritos. Conclui-se que a ferramenta financeira mais utilizada é a técnica do valor presente esperado em sua modalidade de método 2 , para cuja construção se emprega o "modelo de valoração de ativos de capital" a fim de obter a taxa de desconto.

Palavras chave: Valor presente, Valor razoável, NIIF 13, Fluxos de efetivos futuros, Taxa de descontó.

Castellanos, H. (2014) Aplicación Contable del Valor Presente en el Contexto Venezolano. En: Revista de la Facultad de Ciencias Económicas de la Universidad Militar Nueva Granada. rev.fac.cienc. econ, XXII (1).

JEL: M40, M41, M49.
\end{abstract}

\section{Preliminares}

La ciencia contable ha experimentado, en la última década, un cambio de paradigma en la valoración de los elementos que conforman los estados financieros. El criterio valorativo responsable de esta transición es el valor razonable.

Los principales organismos reguladores del área contable a nivel global se han caracterizado por una acentuada incorporación de la acepción del valor razonable en sus respectivos marcos normativos. En la actualidad, el International Accounting Standards Board $^{1}$ (IASB), importante organismo emisor de estándares contables, emplea esta pauta valorativa, de forma obligatoria u opcional, tanto en la valoración inicial como posterior de un número considerable de partidas contables.
Al respecto, Castellanos (2012, 266), en una investigación previa, al analizar la presencia del valor razonable en la NIIF para las PYMES expresó:

El valor razonable tiene un importante protagonismo en la NIIF para las PYMES (2009) afianzándose como su principal criterio valorativo de referencia. Esta afirmación se sustenta en que de las treinta $y$ cinco (35) secciones que posee la norma, el valor razonable es mencionado en veintiséis (26) de ellas, lo cual representa $74,26 \%$ del total del contenido del marco regulatorio. Asimismo, en dieciocho (18) de las secciones, el valor razonable se dispone como la base de valoración obligatoria u optativa para cada una de esas partidas.

1 Traducción al castellano: "Consejo de Normas Internacionales de Contabilidad". En adelante IASB por sus siglas en inglés. 
El avance más significativo, en materia normativa, sobre la aplicación del valor razonable fue la emisión en 2011, por parte del IASB, de la Norma Internacional de Información Financiera ${ }^{2}$ (NIIF) 13 "Medición del Valor Razonable". Con anterioridad a esta regulación, los aspectos que caracterizaban a las mediciones a valor razonable se encontraban dispersos a lo largo de las normas del IASB. De hecho, el diseño de la NIIF 13 se abocó a "reemplazar toda orientación existente sobre la medición hecha a valor razonable contenida en la literatura IFRS, en un solo estándar" (Deloitte, 2009, 1).

El valor razonable es conceptualizado en la NIIF 13 (IASB, 2012a) específicamente, párrafo 24, como: "El precio que se recibiría por la venta de un activo o se pagaría por la transferencia de un pasivo en una transacción ordenada en el mercado principal (o más ventajoso) en la fecha de la medición en condiciones de mercado presentes (es decir, un $\mathrm{p}$ recio de salida) independientemente de si ese precio es observable directamente o estimado utilizando otra técnica de valoración".

Con el objetivo de acrecentar la calidad de la información financiera elaborada a partir de mediciones valoraciones a valor razonable, la NIIF 13 (IASB, 2012a) establece una jerarquía estructurada con una secuencia lógica, compuesta por tres niveles de variables, los cuales se describen a continuación:

- Variables de Nivel 1: caracterizadas por precios cotizados (sin ajustar) para activos o pasivos idénticos a los que posee la entidad. En la fecha de la valoración deben converger condiciones óptimas que garanticen a la entidad el acceso a los valores de cotización.

- Variables de Nivel 2: hacen referencia a precios que pueden o no ser cotizados y a variables observables, de manera directa o indirecta, como tasas de interés, curvas de rendimiento, diferenciales de crédito, entre otras; concernientes a activos y/o pasivos similares ó idénticos a los que tiene la entidad y, que provienen de transacciones recientes. Estos importes pudiesen ser ajustados si, entre otros factores, la gerencia considera que las circunstancias económicas en las que se efectuó la transacción original han experimentado cambios significativos, ó si ha transcurrido un tiempo importante entre la fecha original y el momento sobre el que se informa.

- Variables de Nivel 3: identificadas como no observables o no disponibles en el mercado de comercialización del activo y/o pasivo. Para obtener un valor razonable a partir de variables no observables se recurrirá a técnicas financieras, con el fin de simular los supuestos o hipótesis que los participantes en el mercado usarían para establecer el precio del elemento.

La conformación de la jerarquía del valor razonable supone que, sólo se puede recurrir, en la determinación del importe, a las variables de nivel inferior cuando se hace impracticable o no razonable el uso de variables de un nivel superior. Con el fin de ilustrar la jerarquía aplicable en la medición a valor razonable se presenta un diagrama de flujo en la Ilustración 1.

Al hilo de esta normativa han surgido importantes debates sobre el grado de subjetividad que presenta cada nivel de variables de la jerarquía del valor razonable. A tal efecto, académicos como David (2010), Marchese (2010), Morales (2010), Muñoz (2009) y García \& Zorio (2002) opinan que la subjetividad se incrementa al utilizar variables de niveles inferiores.

Así pues, si el activo, pasivo o patrimonio cuenta con precios cotizados, comercializados en un mercado activo y profundo, la valoración es más fiable, inclusive Muñoz (2009) afirma que estos son valores

\footnotetext{
En adelante NIIF por sus siglas en castellano.

Conviene precisar que en 2006, el Financial Accounting Standards Board (FASB) emitió el SFAS 157 "Mediciones Hechas a Valor Razonable", norma norteamericana pionera en unificar en un solo estándar la regulación del valor razonable.

4 Para un mayor grado de detalle consultar los párrafos del 72 al 90 de la NIIF 13 (IASB, 2012A).
} 
objetivos y verificables. Por el contrario, si la gerencia acude a valorar el elemento a través de técnicas financieras (el nivel más inferior) el importe resultante estará impregnado de un mayor grado de subjetividad. Sobre este particular, Morales $(2010,88)$ al comentar sobre la valoración de instrumentos financieros, expresa lo siguiente:

El problema surge fundamentalmente cuando se han de valorar instrumentos financieros para los que no existe un mercado líquido (no hay precios cotizados). En este caso, la entidad tiene que utilizar modelos de valoración incorporando inputs a veces no observables, con lo cual la verificabilidad es más difícil y la fiabilidad de las estimaciones disminuye.

llustración 1. Jerarquía del valor razonable ${ }^{5}$
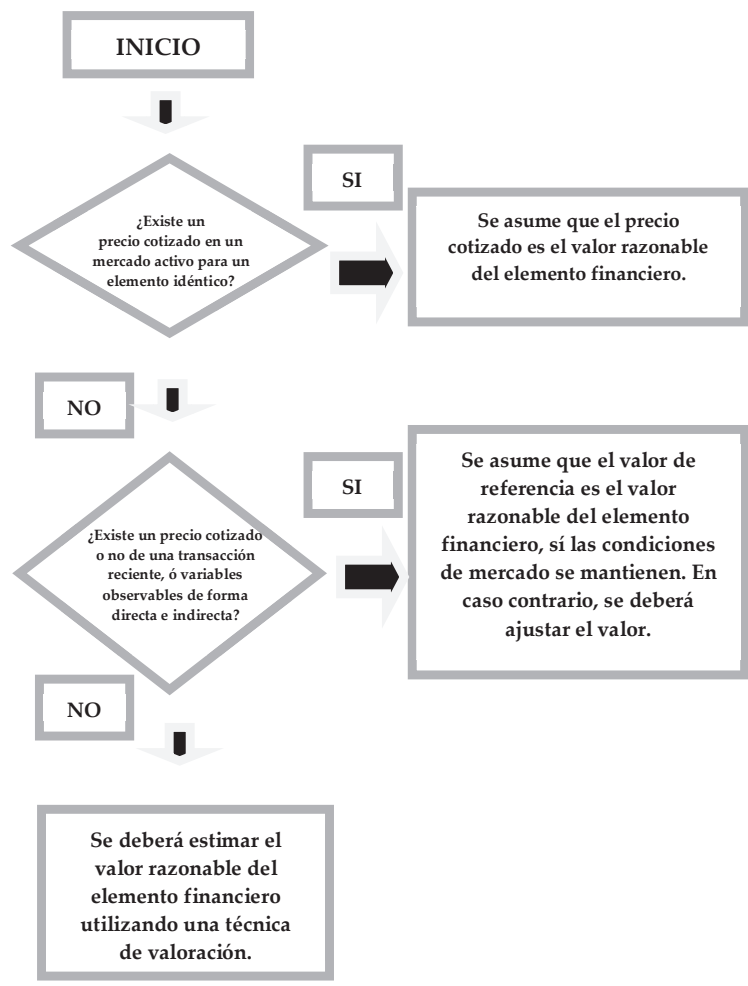

Como se puede inferir, los tratadistas citados en epígrafes anteriores poseen una postura orientada a conectar de forma proporcional el nivel de la jerarquía con el grado de subjetividad. No obstante, en opinión del investigador estos argumentos son discutibles, ya que, ningún nivel jerárquico está exento a ser subjetivo, aún más si se examinan los factores económicos en Venezuela ${ }^{6}$, caracterizados por una excesiva volatilidad, elevada inflación, disparidad colosal entre la tasa de cambio de la divisa oficial y la paralela o no oficial, disminución significativa de la oferta de bienes y servicios, aumento considerable de las importaciones, inseguridad jurídica, cierre de casas de bolsa, entre otros aspectos. Ahora bien, al estudiar la subjetividad presente en el primer nivel de la jerarquía, Valle $(2009,147)$ indica que:

\section{Las cotizaciones bursátiles o los valores por metro cuadrado de las unidades inmobilia- rias negociadas en un área específica de la ciudad, los mismos tienen un fuerte com- ponente de subjetividad derivado de las ex- pectativas de los agentes económicos que pueden verse afectadas por circunstancias como rumores del mercado, expectativas o ruido político que en muchos casos origi- nan una alta volatilidad de los precios de mercado.}

En virtud de lo antes expuesto se podría afirmar que, a pesar de que un elemento financiero se comercialice en un mercado activo, una bolsa de valores, por ejemplo, sus cotizaciones no aseguran que sean el fiel reflejo de sus valores razonables. Es así como al analizar la actividad de la Bolsa de Valores de Caracas (BVC), principal mercado bursátil venezolano, se deduce que es una opción cada vez menos atractiva para los inversionistas. Sobre este particular, en una entrevista publicada en el sitio web guía.com.ve en el año 2009, Nelson Ortiz, ex presidente de la BVC, explica:

Se ha convertido en un mercado con poca profundidad, donde se efectúan escasas

\footnotetext{
Fuente: Elaboración propia.

Venezuela adoptó con reservas el marco contable del IASB en 2008 para grandes entidades y en 2011 para PYMES.
} 
transacciones. Con el volumen que maneja diariamente, es una de las menos importantes de América Latina, región donde históricamente era una de las más relevantes hasta el momento en que llegó la crisis financiera en la década de los 90. Luego de eso, ha tenido sus momentos pico y sus etapas difíciles.

Otro mercado financiero que no ha logrado consolidarse en Venezuela, es la Bolsa Pública de Valores Bicentenaria (BPVB), creada el 13 de noviembre de 2010 mediante Gaceta Oficial Extraordinaria N ${ }^{\circ}$ 5.999, con el propósito de comercializar títulos valores emitidos por "los entes públicos, las empresas públicas, las empresas del Estado, las empresas de propiedad social o colectiva, las empresas mixtas, las cajas de ahorro de los entes públicos, las comunidades organizadas, institutos autónomos, las empresas privadas, las pequeñas y medianas empresas, la República Bolivariana de Venezuela" (Ley de la Bolsa Pública de Valores Bicentenaria, 2010).

Así lo corrobora, la información suministrada por la Agencia Venezolana de Noticias (AVN) en su sitio web, en fecha 09 de enero de 2012:

En su primer año de funcionamiento, la Bolsa Pública de Valores Bicentenaria hizo 157 operaciones de deudas públicas y privadas, con colocaciones de deudas de Títulos de Empresas Privadas por 273 millones de bolívares, todos aprobados por la Superintendencia Nacional de Valores.

Tal y como se ha observado, los principales mercados de capitales en Venezuela son poco dinámicos, causando dudas sobre la actividad o inactividad de ellos. Una aproximación a esta aserción, la expone el Panel Asesor Experto del IASB (2008, 14), en su documento intitulado "Medición y revelación del valor razonable de instrumentos financieros en mercados que ya no están activos", al indicar que una característica, no limitativa, que podría identificar si un mercado presenta inactividad está vinculada con "una declinación importante en el volumen y en el nivel de la actividad de negociación".
En relación a la obtención de valores razonables de activos no financieros, como propiedades de inversión, a partir de los valores asignados por metro cuadrado a áreas concretas de un determinado sector, mencionado por Valle (2009), se puede puntualizar que, el mercado inmobiliario en Venezuela, caracterizado por el alza sostenida del costo de estas propiedades, dificulta un óptimo proceso de valoración que se sustente, únicamente, en valores de referencia. Es común toparse en la prensa escrita venezolana con reseñas como "el mercado inmobiliario no recobra la normalidad, si se puede usar esta palabra en un sector que ha estado afectado en los últimos años por un sinnúmero de leyes, expropiaciones, intervenciones e invasiones" (Rojas, 2013).

En este sentido, en una entrevista concedida al diario El Universal, Magaly Varela (2013), presidenta de la Cámara Inmobiliaria Metropolitana, señaló que "las viviendas que están en el mercado aumentan aceleradamente sus precios y esto es producto, fundamentalmente, de la insuficiente cantidad de viviendas en oferta y de la devaluación de la moneda".

Resulta lógico, a la luz de las particulares de la economía venezolana, que al supeditar el proceso de valoración a valor razonable al primer nivel de variables el componente subjetivo, en una alta proporción, estará presente. Por su parte, el segundo nivel de variables de la jerarquía del valor razonable eleva la incertidumbre que rodea al proceso de valoración. En este nivel jerárquico, la gerencia de la entidad debe emplear su juicio profesional para examinar si, los precios de referencia de transacciones recientes de elementos idénticos o similares cotizados en mercados activos o inactivos y, las variables observables tienen relación directa con el elemento financiero a valorar en el presente.

De tal manera que, cuando los precios de referencia distan de los valores razonables, la gerencia debe ejecutar un proceso de ajuste para incorporar al importe resultante los cambios suscitados en las condiciones económicas del entorno de la entidad. En caso de que los ajustes sean significativos, el proceso de valoración migra al siguiente nivel de variables, es decir, al nivel tres de la jerarquía. 
Conviene resaltar que, el enfoque regulador del IASB no precisa procedimientos específicos para realizar los ajustes, lo que incrementa la subjetividad, al dejar a la gerencia una absoluta discrecionalidad al momento de valorar. En esta línea, la NIIF 13 (IASB, 2012a)en su párrafo B39 indica "esta NIIF no prescribe una metodología para realizar ajustes significativos a las transacciones o precios cotizados".

Al analizar el segundo nivel de la jerarquía, la prestigiosa firma auditora PricewaterhouseCoopers (2009, 9) recomienda:

Teniendo en cuenta el cambio significativo en las condiciones del mercado, no creemos que el uso de referencias de precios recientes (por ejemplo, de los últimos doce meses) sean necesariamente aplicables, puesto que pueden no reflejar las condiciones actuales de mercado. Por lo tanto, dado el clima económico actual, es recomendable revisar las circunstancias de cada transacción antes de utilizar el precio pagado como referencia.

Al observar los factores que caracterizan al segundo nivel de variables y al vincularlo con las propiedades del sistema económico venezolano, el investigador infiere que los ajustes, en la mayor parte de los casos, serán significativos.

Luego de contrastar los dos primeros niveles de la jerarquía del valor razonable con las condiciones económicas en Venezuela, el investigador concluye que, por ausencia de mercados activos y por la necesidad de realizar ajustes significativos a los precios de transacciones recientes, la valoración de transacciones a valor razonable en Venezuela estará circunscrita, mayoritariamente, a variables del tercer nivel.

La aplicación de técnicas financieras expuesta por el IASB, propias del tercer nivel de variables, muestra una especial predilección por el uso de la técnica de valor presente. A tal efecto, el Panel Asesor Experto del IASB (2008) puntualiza, en su párrafo 84, "una técnica de valuación comúnmente usada es el modelo de flujos de efectivo descontados". Por su parte, la NIIF 13 (IASB, 2012a), a pesar de no indicarlo de forma expresa, dedica 21 párrafos $^{7}$ de su guía de aplicación para explicar detalladamente la utilización de la técnica del valor presente.

Al igual que los dos primeros niveles de variables, el tercer nivel también está inmerso en un contexto subjetivo. En este sentido, el proceso de construcción de los inputs que conforman la técnica financiera del valor presente contiene implícita una marcada discrecionalidad, debido a que la gerencia participa en la emisión de un número importante de juicios encaminados a seleccionar, entre otros aspectos, la tasa de descuento y los flujos de efectivo a descontar.

Finalmente, al suponer que en Venezuela gran parte de las mediciones a valor razonable se realizan en atención al tercer nivel jerárquico y que, en este nivel de variables la técnica financiera de mayor representatividad es el valor presente, la inquietud de investigación se relaciona con saber ¿Cuáles son los criterios utilizados por los Contadores Públicos para aplicar el valor presente en el marco del contexto venezolano?

\section{Metodología}

El objeto de estudio del presente trabajo investigativo son los criterios que utilizan los Contadores Públicos en la aplicación del valor presente en Venezuela. En esta investigación de tipo analítica se procedió, inicialmente, a realizar una revisión documental de la NIIF 13 (IASB, 2012a), norma contable que establece el marco para la medición del valor razonable. Seguidamente, se incorporó los resultados de una encuesta ${ }^{8}$ realizada a un grupo de doce Contadores Públicos que laboran en reconocidas firmas de auditoría del país ${ }^{9}$ y que ostentan un cargo no inferior a supervisor ${ }^{10}$.

Para obtener una mayor información consultar del párrafo B12 al B33 de la guía de aplicación de la NIIF 13 (IASB, $2012 a)$.

8 Conviene destacar que la técnica de recolección de datos que apoyó el presente estudio cualitativo es la encuesta, diseñada sólo con preguntas abiertas. La explicación radica en que el investigador, únicamente, tuvo contacto con los informantes claves a través de correo electrónico, por lo que no logró conocer expresiones lingüísticas o corporales que sí serían vinculantes en una entrevista.

9 Se consultaron Contadores Públicos que trabajan en: Espiñeira, Pacheco \& Asociados; Rodríguez, Velázquez \& Asociados; Marambio, González \& Asociados; y Llobet, Lugo \& Asociados.

10 Para alcanzar el cargo de supervisor el tiempo promedio de permanencia en la firma es de cuatro años. 
En virtud de que, el presente trabajo investigativo se desarrolla en atención a la NIIF 13 (IASB, 2012a), el investigador infiere que la opinión de profesionales que pertenecen al capital humano de prestigiosas firmas auditoras que, en su mayoría, representan a firmas internacionales, por estar en una constante actualización, contribuye significativamente al éxito de la investigación. La escogencia de los informantes claves que se encuestaron fue determinada por contactos de referencia en las firmas, quienes luego de recibir una comunicación del investigador remitieron el correo electrónico de varios de sus supervisores. Estos profesionales completaron el cuestionario vía online.

El cuestionario presenta un conjunto de seis preguntas de carácter abierto, por lo que no existe limitante para la opinión de los expertos. Las interrogantes del instrumento de recolección de datos están encaminadas a conocer: las categorías de los elementos financieros que se miden a valor presente, la clase de técnica de valor presente a utilizar, la metodología utilizada para obtener la tasa de descuento y los flujos de efectivo futuros.

Para el análisis e interpretación de la información obtenida, el investigador consideró el uso del análisis de contenido y del análisis hermenéutico, con el propósito de aproximarse al verdadero significado de las palabras contenidas en los textos que abordan el tema del valor presente y, de las expresadas por los expertos en sus correspondientes encuestas. Por su parte, la validez del instrumento de recolección de datos se alcanzó con la revisión de tres expertos, el primero relacionado con el área de contenido, específicamente en las NIIF (2012), el segundo es destacado en el campo de la metodología y, el tercero inmerso en el área de lengua y redacción.

\section{El valor presente en la NIIF 13 (IASB, 2012a)}

El tercer nivel de la jerarquía contempla el uso de técnicas financieras para la determinación del valor razonable. En este sentido, la NIIF 13 (IASB, 2012a), en su párrafo 62 , razona sobre la utilización de técnicas de valoración, "El objetivo de utilizar una técnica de valoración es estimar el precio al que tendría lugar una transacción ordenada de venta del activo o de transferencia del pasivo entre participantes de mercado en la fecha de la medición en las condiciones de mercado presentes".

Asimismo, la disposición normativa de la NIIF 13 (IASB, 2012a) clasifica las técnicas financieras de acuerdo al enfoque de valoración. Las categorías a saber son: enfoque de mercado, enfoque del costo y enfoque del ingreso. La aplicación del valor presente a las categorías de activos $y / o$ pasivos mencionados por los expertos es compatible con el marco normativo del IASB.

Dentro del enfoque del ingreso se encuentra la técnica financiera del valor presente, caracterizada por "convertir importes futuros (por ejemplo, flujos de efectivo o ingresos y gastos) en un importe presente único (es decir, descontado)" (IASB, 2012a, parr. B10).

\subsection{Principios generales aplicables al valor presente}

En el párrafo B14 de la NIIF 13 (IASB, 2012a) se esgrimen un conjunto de principios generales que rigen la aplicación de la técnica de valor presente, los cuales se describen a continuación:

(a) Los flujos de efectivo y tasas de descuento deberían reflejar los supuestos que los participantes de mercado utilizarían al fijar el precio del activo o pasivo. (b) Los flujos de efectivo y las tasas de descuento deberían tener en cuenta solo los factores atribuibles al activo o pasivo que se está midiendo. (c) Para evitar la doble contabilización o la omisión de los efectos de los factores de riesgo, las tasas de descuento deberían reflejar supuestos que sean coherentes con los inherentes a los flujos de efectivo. (d) Los supuestos sobre flujos de efectivo y tasas de descuento deben ser coherentes internamente.

Como se puede apreciar, los principios generales aplicables a la técnica de valor presente están orientados a que las variables (flujos de efectivo y tasa de descuento), asociadas a la determinación del precio 
de un bien o servicio, representen fielmente las expectativas de los participantes en el mercado. Resulta oportuno indicar que las variables deben incluir los factores de riesgo inherentes a la transacción, en virtud de que, al no incorporarse el componente de riesgo el importe resultante, según la norma contable ${ }^{11}$, no se considerará como razonable.

Un aspecto a mencionar se vincula con la correspondencia de los flujos de efectivo y la tasa de descuento con el elemento a valorar. En este punto es necesario precisar que los flujos de efectivo se construirán atendiendo a los derechos u obligaciones contractuales de cada activo y/o pasivo, la capacidad normal de producción de cada entidad, la vida útil y valor de salvamento de cada activo, entre otros factores. Por su parte, la tasa de descuento a emplear dependerá de las características de cada transacción, ya que, a título de ejemplo, la tasa de descuento de un instrumento financiero básico no necesariamente deberá coincidir con la de una propiedad de inversión. En todo caso, la tasa de descuento para un instrumento financiero básico puede obtenerse a través de la comparación con las tasas de rendimiento, observables en el mercado, de instrumentos similares o equivalentes, mientras que para una propiedad de inversión podría acudirse a una tasa libre de riesgo, por citar alguna opción. En síntesis, las variables se estructuran de acuerdo a los atributos específicos de los elementos sometidos al proceso de valoración, por lo que no existen parámetros rígidos o de aplicación universal para la construcción de las variables.

El principio general encaminado a impedir la doble incorporación de los factores de riesgo o, en contraste, su omisión, apuntala a alcanzar un equilibrio en el componente de riesgo adherido a las variables de flujos de efectivo y tasa de descuento. Así, por ejemplo, si los flujos de efectivo vinculados a un elemento financiero contienen los niveles de incertidumbre sobre incumplimientos futuros, es decir, se encuentran ponderados en atención a la probabilidad de ocurrencia de ciertos eventos, la tasa de descuento a aplicar debe prescindir de incluir el riesgo de incum- plimiento, debido a que en el valor final convergería, doblemente, un mismo tipo de riesgo.

El último de los principios que sustentan la aplicación del valor presente explica la coherencia que debe existir al relacionar las variables que intervienen en la valoración. En este contexto, si los flujos de efectivo se estructuran, por ejemplo, sobre la base de moneda corriente, la tasa descuento respectiva también deberá contener los efectos de la inflación.

\subsection{Los componentes de la técnica de valor presente}

Los componentes de la técnica financiera de valor presente descritos en el párrafo B13 de la NIIF 13 (IASB, 2012a) se pueden sintetizar en tres elementos a saber: estimación de los flujos de efectivo esperados, determinación de la tasa de descuento e inclusión de una prima de riesgo a la valoración.

En todo proceso de valoración a valor presente, la gerencia de la entidad deberá formular supuestos internos que permitan estructurar los posibles flujos de efectivo que se derivarán de la tenencia y/o uso del elemento contable. Estas hipótesis considerarán cualquier incertidumbre asociada a la cantidad, fluctuación y oportunidad de los flujos de efectivo futuros.

Otro elemento que resalta en las valoraciones a valor presente, es el vinculado a la tasa de descuento que se incluirá al modelo. Esta tasa representa el efecto del valor del dinero en el tiempo. Generalmente, se emplea una tasa de interés pura o libre de riesgo, relacionada con un activo y/o pasivo cuyos flujos de efectivo sean coherentes con los del elemento a valorar.

Un factor clave en la determinación del valor presente es la incorporación del componente de riesgo, incluidos, si aplican, los riesgos por exposición al mercado, los riesgos de crédito de la contraparte y los riesgos de crédito propio. En este sentido, la prima de riesgo que se adhiere al proceso de valoración podrá ser parte integrante de los flujos de efectivo esperados o de la tasa de descuento.

11 Para profundizar consultar el párrafo 88 de la NIIF 13 (IASB, 2012A). 


\subsection{Diversidad de técnicas de valor presente}

La técnica de valor presente pretende lograr que los desembolsos y/o ingresos de efectivo futuros, asociados a un elemento contable, confluyan en un importe actual único. Para alcanzar este objetivo y, considerando la gran diversidad de activos y pasivos que son susceptibles a ser valorados a través de esta técnica financiera, existen distintas variaciones de la técnica la técnica de valor presente.

Al respecto, la NIIF 13 (IASB, 2012a) aclara, en su párrafo $\mathrm{B} 12$, que su guía de aplicación "ni prescribe el uso de una técnica de valor presente específica única ni limita el uso de las técnicas de valor presente para medir el valor razonable". Por lo tanto, el preparador de la información financiera deberá encausar su juicio profesional a determinar ¿cuál es la técnica de valor presente que mejor se adapta a los atributos o propiedades del activo o pasivo sometido a valoración?

Las técnicas de valor presente mencionadas en el párrafo B17 de la NIIF 13 (IASB, 2012A)son: técnica de ajuste de la tasa de descuento y técnica del valor presente esperado.

\subsubsection{Técnica de ajuste de la tasa de descuento}

La aplicación de la técnica de ajuste de la tasa de descuento se inicia con la estructuración de los flujos de efectivo esperados del elemento a valorar. Estos flujos son el resultado de un proceso de estimación que considera una prima de riesgo, ya que, a pesar de que los flujos de efectivo futuros de un elemento contable estén definidos contractualmente, su materialización está supeditada a la realización de ciertos eventos. Tal es el caso de una inversión en un instrumento de deuda de renta fija, en el que el tenedor del instrumento deberá incorporar el riesgo de crédito a la valoración.

El Panel Asesor Experto del IASB (2008) puntualiza que la cuantificación del riesgo de crédito se deriva de la evaluación de la posición financiera del emisor y de los activos que proveen respaldo al crédito. En todo caso, el examinar la calidad del activo colateral que da soporte financiero al instrumento de deuda se convierte en un factor determinante en el proceso de valoración. Partiendo de esta premisa, el Panel Asesor Experto del IASB $(2008,18)$ concluye que "a mayor sea el valor y la calidad de los activos a los cuales la entidad tenga recurso en el evento de incumplimiento, menor es el riesgo de crédito del instrumento". En suma, se requiere, como paso previo, examinar los posibles flujos de efectivo del activo colateral para estimar los flujos de efectivo contractuales del instrumento de deuda.

Luego de evaluar el riesgo de crédito inherente al elemento contable, se procede a analizar los componentes y particularidades de los activos o pasivos similares que se encuentran disponibles en el mercado. Este examen comprende la naturaleza de los flujos de efectivo esperados, su grado sensibilidad ante los cambios en las variables económicas, la posición crediticia de la entidad emisora, las garantías que respaldan a los activos en estudio, entre otros factores. El resultado de este proceso deberá indicar qué activo o pasivo se compara, en mayor medida, con el elemento de la entidad que se somete a valoración.

El objetivo de buscar un activo o pasivo comparable se orienta a la determinación de su tasa implícita de rendimiento, para posteriormente aplicar esta tasa, por analogía, en el descuento del elemento de la entidad.

\subsubsection{Técnica del valor presente esperado}

La aplicación de la técnica del valor presente esperado exige el cómputo de los flujos de efectivo probables que se asocien al activo o pasivo. Esto se logra con el diseño de los posibles escenarios que ocasionarán flujos de efectivo y su correspondiente probabilidad de ocurrencia. "En términos estadísticos, es el promedio ponderado de los valores posibles de una variable aleatoria discreta con las probabilidades respectivas como ponderaciones" (IASB, 2012a, párr. B23).

Para ilustrar la determinación de los flujos de efectivo probables ponderados se tiene un activo que posee asociado dos potenciales desenlaces al cabo de un año. El primer escenario plantea que los flujos de 
efectivo posibles que generará el activo ascienden a 40.000 u.m. con una probabilidad de ocurrencia del $90 \%$, mientras que, el segundo escenario propone que el activo generará flujos de efectivo posibles por un monto de 50.000 u.m. con una probabilidad de ocurrencia del $10 \%$. En consecuencia, los flujos de efectivo probables ponderados son de $41.000^{12} \mathrm{u} . \mathrm{m}$.

Ahora bien, al medir a valor razonable un elemento contable, a partir de la técnica de valor presente esperado, se pueden emplear dos métodos. El uso de uno de ellos en detrimento de otro dependerá, exclusivamente, de la disponibilidad de los datos, las características del elemento a valorar y del juicio profesional.

\subsubsection{Técnica del valor presente esperado método 1}

La técnica del valor presente esperado en su método 1 incorpora al modelo valorativo el riesgo sistemáti$\mathrm{CO}^{13}$ para ajustar los flujos de efectivo probables ponderados, obteniendo un flujo de efectivo equivalente cierto. Para ilustrar este enunciado se presenta el siguiente ejemplo, una entidad estimó que uno de sus activos era capaz de generar un flujo de efectivo probable ponderado de 2.000 u.m. al año. Además conoce que, un activo similar tiene una prima de riesgo sistemática o de mercado que asciende al $5 \%$. Ante tal escenario, el flujo de efectivo equivalente cierto es de $1.900^{14}$ u.m. Finalmente, para el cálculo del valor presente se descuenta el flujo de efectivo equivalente cierto a una tasa de interés libre de riesgo.

Conviene precisar que, la técnica del valor presente esperado en su método 1 sólo incluye la prima de riesgo sistemática en los flujos de efectivo probables ponderados y, en ningún momento, el riesgo forma parte integrante de la tasa de descuento. Esta aserción da sustento al principio general aplicable al valor presente orientado a evitar la doble contabilización de los efectos de los factores de riesgo.

\subsubsection{Técnica del valor presente esperado método 2}

La técnica del valor presente esperado en su método 2 considera el riesgo sistemático para ajustar la tasa de descuento libre de riesgo. En consecuencia, el valor presente se determina al descontar los flujos de efectivo probables ponderados a una tasa de interés conformada por una tasa libre de riesgo y una prima de riesgo de mercado.

En virtud de lo antes expuesto, los flujos de efectivo probables ponderados no se ajustan en atención al riesgo sistemático, en contraste, sólo se ajusta la tasa de descuento.

\section{Criterios contables utilizados por expertos para la aplicación del valor presente en Venezuela}

Al consultarles a los expertos acerca de la frecuencia en que emplean o auditan la técnica de valor presente en la determinación del valor razonable de los elementos de los estados financieros, resalta el hecho de que un $100 \%$ contestó que usa o audita la técnica de valor presente con alta frecuencia. Conviene puntualizar que, un $25 \%$ de los encuestados manifestaron el alto grado de complejidad que supone trabajar con esta técnica financiera. En la Tabla 1 se muestran las respuestas de los encuestados.

Los resultados obtenidos en la encuesta son coherentes con la propensión de los organismos emisores a la aplicación de la técnica de valor presente. Esta aseveración soporta lo expuesto por el Panel Asesor Experto del IASB (2008) en su párrafo 84 y por el marcado énfasis que realiza la NIIF 13 (IASB, 2012a), en su guía de aplicación, al emplear la cantidad de 21 párrafos, los cuales representan el $45 \%$ del total de los párrafos que componen tal disposición, para la explicación y detalle de la técnica financiera del valor presente.

12 Este monto se obtiene de la siguiente operación matemática: $=(40.000 * 90 \%)+(50.000 * 10 \%)=41.000$ u.m.

13 "Es el riesgo común compartido por un activo o un pasivo con los otros elementos de una cartera diversificada" (IASB, 2012a, párr. B24).

14 Este monto se obtiene de la siguiente operación matemática: $=2.000-(2.000 * 5 \%)=1.900$ u.m. 
Tabla 1. ¿Con qué frecuencia emplea o audita el valor razonable determinado a través de la técnica de valor presente? ${ }^{15}$

\begin{tabular}{|c|l|}
\hline Encuestado & \multicolumn{1}{|c|}{ Respuesta } \\
\hline 1 & La frecuencia es alta. \\
3 & Muy alta. \\
4 & Alta. \\
& Bastante frecuente. Es una tarea compleja. \\
5 & Es una técnica complicada de auditar por los juicios em- \\
& pleados y los datos del mercado, sin embargo, es usada \\
6 & con mucha frecuencia. \\
7 & Alta frecuencia. \\
8 & Alta. Hoy en día es común conseguir está técnica en la \\
9 & Alta. \\
10 & Muy frecuente. \\
11 & Una alta frecuencia. \\
12 & Alta. \\
\hline
\end{tabular}

Sobre el grado de complejidad que trae consigo la utilización de la técnica de valor presente, David $(2010,91)$ expresa que a menor nivel de la jerarquía mayor será la dificultad de "verificar que los valores razonables calculados no provoquen sobrevaloraciones de activos y/o subvaluaciones de pasivos". Al respecto, Pastor $(2010,85)$ señala que "este criterio de valoración, aunque es directamente relevante para la predicción, es con frecuencia difícil de aplicar a activos y pasivos concretos".

En relación a las clases de activos y/o pasivos que usualmente están valorados a valor presente, los resultados derivados de la encuesta evidencian la supremacía de los instrumentos financieros. A tal efecto, $92 \%$ mencionó a los instrumentos financieros, incluidos, los básicos y los compuestos, $33 \%$ enunció a las provisiones, $33 \%$ se refirió a los ingresos, $25 \%$ listó a los pasivos por beneficios a empleados, $17 \%$ señaló al arrendamiento financiero, y $17 \%$ se refirió al deterioro de valor. El Tabla 2 muestra las respuestas de los expertos consultados.

Tabla 2. ¿Qué grupos financieros usualmente están valorados a partir de la técnica de valor presente ${ }^{16}$

\begin{tabular}{|c|c|}
\hline Encuestado & Respuesta \\
\hline 1 & Instrumentos financieros de renta fija y variable. \\
\hline 2 & $\begin{array}{l}\text { Instrumentos financieros básicos. Instrumentos financie- } \\
\text { ros compuestos. }\end{array}$ \\
\hline 3 & Bonos y papeles comerciales. \\
\hline 4 & $\begin{array}{l}\text { Pasivos por beneficios a empleados, instrumentos finan- } \\
\text { cieros básicos, derivados. }\end{array}$ \\
\hline 5 & $\begin{array}{l}\text { Instrumentos financieros básicos y compuestos, ingre- } \\
\text { sos, planes de pensiones, provisiones, créditos fiscales } \\
\text { por recuperar. }\end{array}$ \\
\hline 6 & Activos financieros y derivados. \\
\hline 7 & $\begin{array}{l}\text { Provisiones por retiros de activos, provisiones por bene- } \\
\text { ficios a empleados, instrumentos financieros, deterioro } \\
\text { de activo, arrendamiento financiero, ingresos diferidos. }\end{array}$ \\
\hline 8 & Instrumentos financieros y provisiones. \\
\hline 9 & Instrumentos financieros básicos y compuestos. \\
\hline 10 & Títulos de deuda y patrimonio. \\
\hline 11 & Provisiones e ingresos con intereses implícitos. \\
\hline 12 & $\begin{array}{l}\text { Arrendamiento financiero, Instrumentos financieros, de- } \\
\text { terioro de valor, ingresos. }\end{array}$ \\
\hline
\end{tabular}

La aplicación del valor presente a las categorías de activos $y / o$ pasivos mencionados por los expertos es compatible con el marco normativo del IASB (2012). Al respecto, la NIIF 9 (IASB, 2012b) "Instrumentos Financieros" puntualiza, en su párrafo 5.1.1, que en el reconocimiento inicial los activos y pasivos financieros se deberán medir a su valor razonable, salvo ciertas excepciones. Ahora bien, para su reconocimiento posterior el criterio del valor razonable, descrito en los párrafos 5.2.1 y 5.3.1, también será aplicable en ambas clases de elementos contables, teniendo la limitación para los pasivos financieros de que su valor razonable no sea inferior al importe necesario para extinguir la obligación ${ }^{17}$. Es preciso explicar que, los créditos fiscales por recuperar, comen-

15 Fuente: Elaboración propia con base a las respuestas de los expertos encuestados.

16 Fuente: Elaboración propia con base a las respuestas de los expertos encuestados.

17 Para obtener una mayor información consultar el párrafo 5.4.3 de la NIIF 9 (IASB, 2012b). 
tados por el encuestado No. 5, simbolizan activos financieros regulados por la NIIF 9 (IASB, 2012b).

Por su parte, la NIC 37 (IASB, 2012g) "Provisiones, Pasivos Contingentes y Activos Contingentes" en su párrafo 45, indica la necesidad de valorar a valor presente las provisiones cuando el valor del dinero en el tiempo sea considerable.

En este orden de ideas, la NIC 18 (IASB, 2012d) "Ingresos de Actividades Ordinarias" establece, en su párrafo 9, que estos beneficios económicos se deben medir al valor razonable de la contrapartida recibida o por recibir. Cabe hacer mención al párrafo 11, el cual revela que, generalmente, la contrapartida toma la forma de efectivo o su equivalente, sin embargo, cuando ésta se difiera en el tiempo, será necesaria la aplicación de una técnica, como el valor presente, con el objeto de reconocer los intereses implícitos en la operación.

Los beneficios a empleados también fueron citados por los expertos, al respecto, la NIC 19 (2012e) "Beneficios a Empleados" señala, en su párrafo 50 sobre pasivos por beneficios definidos y en su párrafo 128 sobre otros pasivos a largo plazo, que su correspondiente medición deberá efectuarse al valor presente de sus obligaciones menos el valor razonable de los activos del plan (de existir activos que financien el plan de beneficios).

En relación al arrendamiento financiero, la NIC 17 (IASB 2012c) "Arrendamientos" explica, en su párrafo 20, que los arrendatarios contabilizarán, esta modalidad de activos y su respectivo pasivo, por un importe que represente el valor razonable del bien arrendado o al valor presente de los pagos mínimos que se derivan del arrendamiento.

En otro eje, los expertos mencionaron al deterioro de valor, siendo éste normado por la NIC 36 (IASB 2012f) "Deterioro del Valor de los Activos". El párrafo 18 exige que el importe recuperable de un activo o de una unidad generadora de efectivo se mida al mayor entre su valor razonable menos los costos de venta y su valor de uso. Como se puede observar, el valor presente puede ser usado de forma reiterada para la determinación del importe recuperable, por una parte, en el cálculo del valor razonable menos los costos de venta y, por otra, en el cómputo del valor de uso. El investigador considera oportuno acotar que, a pesar de que el deterioro de valor no está conllustracion do como una clase de activo o pasivo, es relevante y válido vincularlo a esta discusión, en virtud de la aplicabilidad que posee el valor presente para obtener esta unidad de medida.

Al hilo de esta investigación, se les consultó a los expertos sobre la técnica de valor presente que es más utilizada en los estados financieros que ellos preparan o auditan, siendo sus respuestas las siguientes: $50 \%$ manifestó su preferencia hacia la técnica de valor presente esperado método $2,25 \%$ reveló que sólo existe una técnica de valor presente y, $25 \%$ no comprendió, no sabe o no contestó la interrogante. El Tabla No. 3 muestra los resultados obtenidos.

Es preciso hacer visible que, de las técnicas de valor presente enunciadas por la NIIF 13 (IASB, 2012a), los expertos solo mencionaron al valor presente esperado en su modalidad de método 2 , técnica caracterizada por la estructuración de los flujos de efectivo probables ponderados sin ajustarlos en atención al riesgo sistemático y, en contraste, se incorpora la prima de riesgo sistemática a la tasa de descuento. Resalta en los resultados que ninguno de los expertos mencionó el nombre de la técnica utilizada de manera correcta, lo que evidencia poco dominio conceptual de la NIIF 13 (IASB, 2012a), no obstante, conocen la esencia de la técnica.

Resulta necesario indicar que, tres (3) de los encuestados revelaron la sola existencia de una técnica de valor presente, demostrando con esa afirmación que desconocen la NIIF 13 (IASB, 2012a). Se les solicitó a los expertos que suministraran el criterio o método más utilizado para obtener la tasa de descuento, detectándose los siguientes resultados: $67 \%$ mostró su predilección por el Modelo de Valuación de Activos de Capital (MVAC) ${ }^{18}, 25 \%$ se inclinó por una

18 Original en ingles: "Capital Asset Pricing Model (CAPM)". 
tasa comparativa o tasa implícita de rendimiento de activos similares y el $8 \%$ no dio respuesta a la interrogante. En la Tabla No. 4 se ilustra los criterios señalados.

Tabla 3. ¿Cuál es la técnica de valor presente más utilizada en las valoraciones a valor razonable en los estados financieros que usted prepara o audita? ${ }^{19}$

\begin{tabular}{|c|c|}
\hline Encuestado & Respuesta \\
\hline 1 & El descuento de flujos de efectivo. \\
\hline 2 & $\begin{array}{l}\text { Descontar los flujos estimados de efectivo a una deter- } \\
\text { minada tasa. }\end{array}$ \\
\hline 3 & $\begin{array}{l}\text { No comprendo la pregunta, sólo existe una técnica de va- } \\
\text { lor presente. }\end{array}$ \\
\hline 4 & $\begin{array}{l}\text { Existen varias técnicas, su uso depende de las caracterís- } \\
\text { ticas de lo que se vaya a medir. Lo que se utiliza más es } \\
\text { estimar los flujos de efectivo y descontarlos a una tasa de } \\
\text { interés que contenga un componente de riesgo. }\end{array}$ \\
\hline 5 & $\begin{array}{l}\text { La más usada es la del valor presente esperado con ajuste } \\
\text { de la tasa de descuento por el riesgo. }\end{array}$ \\
\hline 6 & $\begin{array}{l}\text { La técnica de valor presente más utilizada es hacer los } \\
\text { flujos de efectivo que se pueden generar en el futuro, para } \\
\text { luego descontarlo a una tasa de interés que refleja los ries- } \\
\text { gos asumidos por la entidad. }\end{array}$ \\
\hline 7 & $\begin{array}{l}\text { Valor presente esperado descontado con una tasa de des- } \\
\text { cuento que establezca el rendimiento del mercado y una } \\
\text { tasa libre de riesgo. }\end{array}$ \\
\hline 8 & \\
\hline 9 & Sólo hay una única técnica. \\
\hline 10 & $\begin{array}{l}\text { Hay una misma técnica que se aplica a todas las medi- } \\
\text { ciones. }\end{array}$ \\
\hline 11 & $\begin{array}{l}\text { La que busca estimar los flujos de efectivo esperados mul- } \\
\text { tiplicados por su probabilidad de que suceda y desconta- } \\
\text { dos a una tasa de interés que tenga todos los riesgos de la } \\
\text { transacción, como el de mercado y riesgo país. }\end{array}$ \\
\hline 12 & $\begin{array}{l}\text { El método en el que se introduce en la tasa de descuento } \\
\text { el riesgo de mercado, el beta de la industria, y una tasa } \\
\text { pura de interés. }\end{array}$ \\
\hline
\end{tabular}

Como se puede observar, para el cálculo de la tasa de descuento se pueden emplear ciertas herramientas financieras, destacando el MVAC como el método más utilizado por los expertos. El MVAC determina una tasa de retorno que relaciona, de forma proporcional, la prima de riesgo sistemática con el rendimiento del activo, asumiendo que a mayor riesgo de mercado mayor será la retribución.

Tabla 4. ¿Cuál es el criterio o método más utilizado para obtener la tasa de descuento? 20

\begin{tabular}{|c|c|}
\hline Encuestado & Respuesta \\
\hline 1 & Una tasa de interés del mercado. \\
\hline 2 & $\begin{array}{l}\text { La más utilizada es la tasa de interés de un activo similar } \\
\text { y ésta se toma por referencia. }\end{array}$ \\
\hline 3 & $\begin{array}{l}\text { La que más se utiliza es la tasa de interés de un instru- } \\
\text { mento idéntico que esté disponible en el mercado. }\end{array}$ \\
\hline 4 & $\begin{array}{l}\text { El modelo de valuación de activos de capital que inclu- } \\
\text { ye una tasa libre de riesgo, una tasa de rendimiento del } \\
\text { sector. }\end{array}$ \\
\hline 5 & CAPM \\
\hline 6 & $\begin{array}{l}\text { Son muchos los métodos. Considero como el más utili- } \\
\text { zado el modelo de activos de capital que incluye los ries- } \\
\text { gos del mercado, el riesgo país y una tasa libre de riesgo. }\end{array}$ \\
\hline 7 & Capital Asset Pricing Model. \\
\hline 8 & \\
\hline 9 & La técnica que incluye los riesgos. \\
\hline 10 & $\begin{array}{l}\text { El criterio de toma en cuenta todos los riesgos, el del } \\
\text { país, el de la industria y el de mercado. }\end{array}$ \\
\hline 11 & $\begin{array}{l}\text { Es necesario determinar la tasa de descuento con la in- } \\
\text { corporación del riesgo país y del riesgo de mercado. }\end{array}$ \\
\hline 12 & $\begin{array}{l}\text { El método de valuación o equilibrio de activos, este mé- } \\
\text { todo incluye una tasa pura de interés (la tasa de la reserva } \\
\text { federal de los EEUU), un beta del sector } 0 \text { industria y el } \\
\text { rendimiento del sector. }\end{array}$ \\
\hline
\end{tabular}

En relación a la esencia del modelo, Apaza (2008, 120) indica lo siguiente:

Resume un conjunto de predicciones acerca de la relación entre el riesgo de un activo y su rentabilidad esperada de equilibrio. Bajo una serie de supuestos simplificadores, la principal premisa del modelo establece que la prima de riesgo de un activo concreto es proporcional a la prima de riesgo del mercado y al coeficiente beta del valor - relativo al mercado.

19 Fuente: Elaboración propia con base a las respuestas de los expertos encuestados.

20 Fuente: Elaboración propia con base a las respuestas de los expertos encuestados. 
El MVAC requiere la obtención de los siguientes supuestos: una tasa libre de riesgo (por ejemplo, el rendimiento de los bonos de la reserva federal estadunidenses), el beta o nivel de riesgo asociado al sector donde opera la entidad, y el rendimiento esperado del mercado, este último debe incluir, la prima de riesgo del mercado y la tasa de inflación del país de donde se tomó la tasa libre de riesgo, así como el riesgo del país donde participa la entidad. En la Ilustración 2 se muestra la formula de aplicación para la determinación del MVAC.

Ilustración 2. Cálculo del MVAC

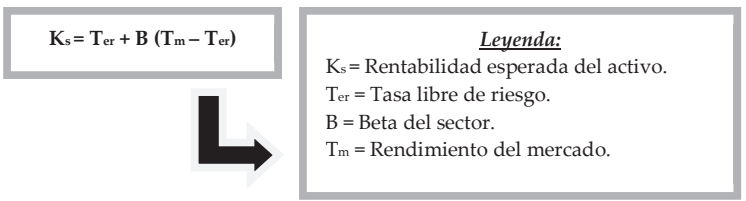

Por otra parte, tres (3) de los expertos consultados enunciaron el criterio de una tasa comparativa o tasa implícita de rendimiento de activos similares. Esta tasa es propia de la técnica de valor presente denominada técnica de ajuste de la tasa de descuento. De tal manera que, el análisis de los componentes y particularidades de los elementos contables similares o idénticos que están disponibles en el mercado juega un rol fundamental para encontrar una tasa de interés por comparación o extrapolación.

Por último, los expertos fueron consultados acerca de las metodologías para la estructuración de los flujos de efectivo futuros y cuál sería un tiempo promedio de proyección para los mismos. Sobre las metodologías para la elaboración de los flujos de efectivo, la encuesta mostró los siguientes resultados: $50 \%$ indicó que los flujos deben ser ajustados por su probabilidad de ocurrencia, $33 \%$ señaló que la formación de los flujos dependerá de las características o atributos de los activos, $8 \%$ reveló que la estructuración de los flujos está sujeta a los riesgos de crédito y, $8 \%$ no contestó. En cuanto al tiempo aproximado de proyección de los flujos, resalta el hecho de que $75 \%$ de los expertos no contestaron y el $25 \%$ restante afirmó que el horizonte de tiempo dependerá de las características del activo. En la Tabla No. 5 se muestran los resultados de la encuesta.

Tabla 5. ¿Cuál es la metodología más utilizada para estructurar los flujos de efectivo futuros? ¿Existe un tiempo promedio de proyección?21

\begin{tabular}{|c|c|}
\hline Encuestado & Respuesta \\
\hline 1 & $\begin{array}{l}\text { No hay metodologías específicas para estimar los flujos de } \\
\text { efectivo, los flujos dependen del activo. }\end{array}$ \\
\hline 2 & $\begin{array}{l}\text { La metodología que generalmente se usa es ajustar los } \\
\text { flujos de efectivo por una tasa que represente el riesgo de } \\
\text { incumplimiento. }\end{array}$ \\
\hline 3 & $\begin{array}{l}\text { No conozco una metodología en particular. Pienso que } \\
\text { dependerá de las propiedades y características del activo. }\end{array}$ \\
\hline 4 & $\begin{array}{l}\text { La metodología más utilizada consiste en calcular la proba- } \\
\text { bilidad de que los flujos de efectivo ocurran o se susciten. } \\
\text { El tiempo promedio de proyección deberá depender del } \\
\text { activo. En Venezuela no debería ser mayor de } 5 \text { años por } \\
\text { las características de la economía. }\end{array}$ \\
\hline 5 & $\begin{array}{l}\text { La que pondera los flujos con su probabilidad para conse- } \\
\text { guir los flujos ponderados. Para esto, se plantean diversos } \\
\text { escenarios con su probabilidad. El tiempo promedio de } \\
\text { proyección depende del activo. }\end{array}$ \\
\hline 6 & $\begin{array}{l}\text { Los flujos de efectivo multiplicados por probabilidad de } \\
\text { ocurrencia. }\end{array}$ \\
\hline 7 & $\begin{array}{l}\text { La metodología a utilizar deberá ponderar los escenarios } \\
\text { posibles por la probabilidad de que estos se den. No existe } \\
\text { un tiempo promedio de proyección. }\end{array}$ \\
\hline 8 & $\begin{array}{l}\text { No creo que existen métodos para estructurar los flujos de } \\
\text { efectivo, los encargados deben estudiar las características } \\
\text { del activo. }\end{array}$ \\
\hline 9 & $\begin{array}{l}\text { Opino que no existen métodos para ello, solo que eso de- } \\
\text { pende del activo. }\end{array}$ \\
\hline 10 & $\begin{array}{l}\text { El método que se utiliza comúnmente es el que incluye la } \\
\text { probabilidad de los flujos. }\end{array}$ \\
\hline 11 & $\begin{array}{l}\text { La que más se utiliza es diseñar un sistema de posibles } \\
\text { desenlaces con sus probabilidades y así obtener flujos } \\
\text { ponderados. Es difícil decir que exista un tiempo promedio } \\
\text { de proyección. }\end{array}$ \\
\hline
\end{tabular}

Como se puede apreciar, los expertos se inclinaron, en su mayoría, por la opción de los flujos de efectivo probables ponderados, lo que es congruente con la técnica del valor presente esperado descrita en el párrafo B23 de la NIIF 13 (IASB, 2012a). Otros in-

21 Fuente: Elaboración propia con base a las respuestas de los expertos encuestados. 
tegrantes del grupo de expertos puntualizaron que la metodología a aplicar dependerá del tipo de activo, siendo esta afirmación, a juicio del investigador, correcta, ya que, en ningún momento, se podrá emplear un método común para todos los activos, porque éstos poseen diversas propiedades o atributos. Asimismo, destaca un experto que comentó acerca del ajuste de los flujos por su riesgo de crédito, esta aserción es válida al equiparla con la técnica de ajuste de la tasa de descuento, la cual expone que ciertos eventos pudieran condicionar la realización de los desembolsos o ingresos de efectivo.

En relación al horizonte promedio de proyección de los flujos de efectivo, los expertos se abstuvieron, en gran medida, de opinar. En tanto que, tres (3) expertos indicaron que este lapso de tiempo obedece al análisis y estudio que se efectúe de cada uno de los activos. Cabe mencionar que, un experto recalcó que en Venezuela no debería realizarse ninguna proyección más allá de cinco (5) años, debido a sus condiciones económicas.

\section{Reflexiones finales}

El valor presente se muestra como la técnica valorativa de mayor importancia en el enfoque regulador para determinar el valor razonable de un elemento contable. Esta aserción está respaldada por el marcado énfasis que realiza la NIIF 13 (IASB, 2012a) en explicar sus principios, componentes, diversidad de técnicas, entre otros aspectos. De la misma manera, los informantes claves manifestaron, por unanimidad, la presencia del valor presente en los estados financieros que ellos han preparado o auditado, principalmente, en la valoración de instrumentos financieros, provisiones, ingresos ordinarios, pasivos por beneficios a empleados, arrendamiento financiera y deterioro de valor.

La técnica de valoración del valor presente está enmarcada en el enfoque del ingreso y apunta a convertir flujos futuros de efectivo en un importe actual único. Para lograr su objetivo, la técnica de valor presente debe incorporar en una de sus principales variables, flujos de efectivo esperados o tasa de descuento, el componente de riesgo que asumen los participantes del mercado, ya que, en caso de no hacerlo, el importe resultante, en atención al marco normativo, carecerá de razonabilidad. Ahora bien, es de considerar la prima de riesgo sólo debe adherirse a una de las variables, de lo contrario se estaría contabilizando de forma doble el riesgo, aminorando la representatividad de las expectativas del mercado.

Un aspecto a mencionar es que, las variables de la técnica de valor presente se deben estructurar en función a los atributos y propiedades particulares de cada elemento contable, siendo improcedente el empleo de una única técnica o herramienta financiera común a todos los activos y/o pasivos a valorar.

La NIIF 13 (IASB, 2012a) revela dos modalidades de la técnica de valor presente, la técnica de ajuste de la tasa de descuento y la técnica del valor presente esperado. Inicialmente, los informantes claves sólo mencionaron a la técnica de valor presente esperado en su método 2. No obstante, en una pregunta posterior, encaminada a conocer la metodología más utilizada para estructurar los flujos de efectivo futuros, las respuestas sobre flujos de efectivo probables ponderados, confirmaron que conocían, la técnica de ajuste de la tasa de descuento. Es preciso resaltar que, los expertos no estaban familiarizados con la terminología que describe la NIIF 13 (IASB, 2012a), ninguno acertó los nombres exactos de las técnicas de valor presente.

En el contexto venezolano, los encuestados indicaron que para la determinación de la tasa de descuento, la técnica financiera más utilizada era el Modelo de Valuación de Activos de Capital, lo que fue coherente con el criterio que enunciaron con anterioridad, en el que prevalecía la técnica del valor presente esperado método 2 . Seguidamente, fue mencionada la tasa comparativa o tasa implícita de rendimiento de activos similares, propia de la técnica de ajuste de la tasa de descuento. Destaca el hecho de que no se señaló al costo promedio ponderado de capital, como técnica financiera para obtener la tasa de descuento.

En este sentido, los resultados de la investigación sugieren que la estructuración de los flujos de efectivo futuros obedece, principalmente, a una metodología que incorpora la probabilidad de que esos desembolsos e ingresos efectivamente ocurran. Nuevamen- 
te, esta afirmación de los expertos coincide con su marcada tendencia a utilizar la técnica de valor presente esperado en su modalidad de método 2.

Para concluir, se propone a la comunidad contable el desarrollo de un trabajo investigativo orientado a conocer, en profundidad, la obtención de la tasa de descuento a partir del Modelo de Valuación de Activos de Capital, enmarcada en las variables económicas de Venezuela, en virtud, de que la presente investigación sólo se limitó a dar a conocer la técnica financiera más utilizada.

\section{Referencias}

Agencia Venezolana de Noticias (2012). Bolsa Pública de Valores Bicentenaria realizó 157 operaciones de deudas públicas y privadas. [Periódico en línea]. Disponible en: http://www.avn. info.ve/contenido/bolsa-p\%C3\%BAblica-valores-bicentenariarealiz\%C3\%B3-157-operaciones-deudas-p\%C3\% BAblicas-yprivadas [Consulta: 2013, Julio 17].

Apaza, M. (2008). Alcances sobre el concepto de valor razonable. En: Revista Internacional Legis Contabilidad \& Auditoría, 36: 89-134.

Castellanos, H. (2012). Estudio Crítico del Valor Razonable. Una Reflexión de su Incidencia en la Calidad de la Información Financiera. Trabajo de grado de maestría no publicado. Mérida: Universidad de los Andes de Venezuela.

David, J. (2010). El valor razonable como criterio básico de medición. [Documento en línea]. Disponible en: http://bibliotecavirtual.unl.edu.ar:8180/publicaciones/bitstream/1/3853/1/ CE_8_1_pag_89_97.pdf [Consulta: 2013, Julio 16].

Decreto con Fuerza dé Ley de la Ley de la Bolsa Pública de Valores Bicentenaria (2010). Gaceta Oficial de la República Bolivariana de Venezuela, 5.999 (Extraordinario), Noviembre 13 de 2010.

Deloitte (2009). Borrador para discusión pública propone orientación ampliada sobre la medición hecha a valor razonable. [Documento en línea]. Disponible en: http:/www.iasplus.com/ espanol/0906fairvaluespanish.pdf [Consulta: 2013, Julio 14].

El Universal (2013). 15\% bajó la oferta de venta de inmuebles en Caracas [Periódico en línea]. Disponible en: http://www. eluniversal.com/economia/130730/15-bajo-la-oferta-de-ventade-inmuebles-en-caracas [Consulta: 2013, Julio 17].

Financial Accounting Standards Board (2006). Statement of Financial Accounting Standards No. 157. [Documento en línea]. Disponible en http://www.fasb.org/cs/ BlobServer?blobcol=u rldata\&blobtable $=$ MungoBlobs\&blobkey $=\mathrm{id} \&$ blobwhere $=1$ 175820927537\&blobheader=application\%2Fpdf [Consulta: 2013, Julio 16].

García, M. \& Zorio, A. (2002). El camino hacia el valor razonable. En: Revista Contabilidad, 5(9): 57-88.

Guía.com.ve (2009). El mercado bursátil venezolano está deprimido y sin dinámica. [Periódico en línea]. Disponible en: http://www. guia.com.ve/noti/34653/el-mercado-bursatil-venezolano-estadeprimido-y-sin-dinamica [Consulta: 2013, Julio 17].
International Accounting Standards Board (2012a). Norma Internacional de Información Financiera $N^{\circ} 13$ Medición del Valor Razonable. Londres, Inglaterra. Traducción oficial el Comité de Revisión de la Fundación IFRS.

International Accounting Standards Board (2012b). Norma Internacional de Información Financiera $N^{\circ} 9$ Instrumentos Financieros. Londres, Inglaterra. Traducción oficial el Comité de Revisión de la Fundación IFRS.

International Accounting Standards Board (2012c). Norma Internacional de Contabilidad $N^{\circ} 17$ Arrendamientos. Londres, Inglaterra. Traducción oficial el Comité de Revisión de la Fundación IFRS.

International Accounting Standards Board (2012d). Norma Internacional de Contabilidad $N^{\circ} 18$ Ingresos Ordinarios. Londres, Inglaterra. Traducción oficial el Comité de Revisión de la Fundación IFRS.

International Accounting Standards Board (2012e). Norma Internacional de Contabilidad $N^{\circ} 19$ Beneficios a Empleados. Londres, Inglaterra. Traducción oficial el Comité de Revisión de la Fundación IFRS.

International Accounting Standards Board (2012f). Norma Internacional de Contabilidad $N^{\circ} 36$ Deterioro del Valor de los Activos. Londres, Inglaterra. Traducción oficial el Comité de Revisión de la Fundación IFRS.

International Accounting Standards Board (2012g). Norma Internacional de Contabilidad No 37Provisiones, Pasivos Contingentes y Activos Contingentes. Londres, Inglaterra. Traducción oficial el Comité de Revisión de la Fundación IFRS.

Marchese, D. (2010). La aplicación del valor razonable (fairvalue) en la medición de los activos biológicos de largo plazo sin mercado activo. En: Revista Información Financiera, Gerencia y Control, 1(1): 13-39.

Morales, J. (2010). ¿Se debería aplicar el valor razonable a todos los instrumentos financieros? En: Revista Española de Financiación y Contabilidad, XXXIX (145): 169-195.

Muñoz, A. (2009). El valor razonable: cautelas y desafíos. En: Revista Intervención General de la Administración del Estado, 22: 48-51.

Panel Asesor Experto del IASB (2008). Medición y revelación del valor razonable de instrumentos financieros en mercados que ya no están activos. [Documento en línea]. Disponible en: http:/ www.iasb.org/NR/rdonlyres/033C2EF4-B437-4AA4-96D11898F8506D7D/0/ExpertAdvisoryGroupReportSpanish.pdf [Consulta: 2013, Julio 20].

Pastor, S. (2010). La Medición de los Activos en las Normas Contables Internacionales. Aplicación del Valor Razonable en la Valuación del Inmovilizado Material. En: Revista de Ciencias Económicas Perspectivas, 2(2): 69-109.

Pricewaterhouse Coopers (2009) Los retos del valor razonable en el entorno de mercado actual. [Documento en línea]. Disponible en: http://kc3.pwc.es/local/es/kc3/ publicaciones.nsf/V1/E06 29150B4F46743C12576CE003873C3/\$FILE/091214\%20 Folleto_Retos\%20valor\%20razonable_final.pdf [Consulta: 2013, Julio 22].

Rojas, I. (2013) Se disparan con el dólar los precios de inmuebles usados. [Documento en línea]. Disponible en: http://www. elmundo.com.ve/ noticias/economia/politicas-publicas/se-disparan-con-el-dolar-los-precios-de-inmuebles-.aspx [Consulta: 2013, Julio 16].

Valle, C. (2009). Hacia el reino de la subjetividad: una crítica al modelo contable del valor razonable. En: Revista Legis de Contabilidad y Auditoría, 438: 133-153. 